\title{
MINI-REVIEW
}

\section{Extracellular Vesicles and Renal Endothelial Cells}

\section{A Fatal Attraction in Hemolytic Uremic Syndrome}

\author{
Elisa Varrone, Domenica Carnicelli, and Maurizio Brigotti
}

From the Department of Experimental, Diagnostic and Specialty Medicine, School of Medicine, University of Bologna, Bologna, Italy

\author{
Accepted for publication \\ February 10, 2021. \\ Address correspondence to \\ Maurizio Brigotti, Ph.D., \\ Department of Experimental, \\ Diagnostic and Specialty Med- \\ icine, University of Bologna, \\ School of Medicine, General \\ Pathology Bldg., Via San Gia- \\ como 14, Bologna 40126, Ita- \\ ly. E-mail: maurizio.brigotti@ \\ unibo.it.
}

\begin{abstract}
This review focuses on typical hemolytic uremic syndrome (HUS), a life-threatening sequela of human infections caused, particularly in children, by Shiga toxin-producing Escherichia coli strains. Thrombotic microangiopathy of the brain and the kidney is the end point of toxin action, resulting in the hallmarks of HUS (ie, thrombocytopenia, anemia, and acute renal failure). A growing body of evidence points to the role of extracellular vesicles released in the blood of patients by toxin-challenged circulating cells (monocytes, neutrophils, and erythrocytes) and platelets, as a key factor in the pathogenesis of HUS. This review provides i) an updated description of the pathogenesis of Shiga toxinproducing $E$. coli infections; ii) an analysis of blood cell-derived extracellular vesicles, and of their parent cells, as triggering factors in HUS; and iii) a model explaining why Shiga toxin-containing vesicles dock preferentially to the endothelia of target organs. (Am J Pathol 2021, 191: 795-804; https://doi.org/10.1016/j.ajpath.2021.02.011)
\end{abstract}

The study of the pathogenesis of a given disease outlines the triggering factors and describes the chain of events involved in the development of the pathologic condition. In the case of infectious diseases, the identification of the microbial pathogenic factors and of the resulting host-pathogen interactions clarifies the mechanisms underlying the pathogenetic process. In many cases, however, a particular disease is the consequence of the complex interactions of several virulence factors, whereby the illness may not occur if one of these factors is removed or if their interplay is impaired. This review focuses on typical hemolytic uremic syndrome (HUS), a life-threatening condition caused by pathogenic bacteria known as Shiga toxin-producing Escherichia coli (STEC), which release potent exotoxin, named Shiga toxins (Stx). ${ }^{1,2}$ The syndrome is the leading cause of acute renal failure in children aged $<3$ years, being characterized also by microangiopathic anemia and thrombocytopenia. $^{1,2}$ HUS can occur in sporadic form, as microepidemics, or as community-wide foodborne outbreaks, as dramatically exemplified in recent years worldwide. ${ }^{1}$

\section{The Basics of the Pathogenesis of STEC Infections}

HUS is a severe sequela of STEC infections occurring after a prodromal intestinal phase, characterized by watery and bloody diarrhea. ${ }^{2}$ STEC are complex pathogens capable of tight and intimate interactions with the gut mucosa, culminating in the derangement of the absorption properties of the enterocytes (watery diarrhea). ${ }^{3}$ These Stx-independent injuries, known as attaching and effacing lesions, are critical for the subsequent pathogenetic steps, such as the synthesis and release of Stx, which cross the intestinal mucosa to reach the lamina propria. ${ }^{2,3}$ Bloody diarrhea is the consequence of toxin actions on the endothelial lining of the intestine, culminating in typical histopathologic changes, such as mucosal and submucosal edema, hemorrhage, focal necrosis, and thrombotic microangiopathy. ${ }^{4,5}$ Lesions to the

\footnotetext{
Supported by the University of Bologna grants Ricerca Fondamentale Orientata (M.B.).

Disclosures: None declared.
} 
microvasculature of the gut trigger bloody diarrhea during precocious toxemia, whereas HUS develops 5 to 7 days later when the endothelial cells of the kidney and brain are targeted by the toxin. ${ }^{2}$ Endothelial damage/dysfunction related to Stx action causing thrombotic microangiopathic lesions in target organs is considered the major pathogenetic event in HUS and occurs when Stx cross the epithelial lining of the large bowel and are transported in circulation. These injuries narrow renal glomerular capillaries, thereby damaging passing erythrocytes, and consume platelets; therefore, acute renal failure, thrombocytopenia, and anemia ensue. $^{1,2}$ The latter condition is exacerbated by the specific targeting of red cell precursor by $\mathrm{Stx}^{1}$ and by complementmediated Stx-triggered hemolysis of mature erythrocytes. ${ }^{6}$

Stx are a family of AB5 bacterial toxins consisting of two main types (Stx1 and Stx2) and many different subtypes (4 for Stx 1 and 12 for Stx2). ${ }^{1,7,8}$ Only a few of these have been found in STEC isolates associated with severe human illness [eg, Stx1a (bloody diarrhea and rarely HUS) or Stx2a, mucus-activatable Stx2d, and the controversial Stx2c (bloody diarrhea and HUS)]. ${ }^{8}$ Stx2a is mainly involved in HUS because the STEC strains that produce and release this subtype are epidemiologically associated with HUS. ${ }^{1,9}$

Stx are rarely found free in the bloodstream of patients during HUS ${ }^{10-14}$; rather, they are found free in blood during late toxemia and before the onset of HUS, as well as associated with circulating cells (neutrophils, monocytes, and erythrocytes) and platelets..$^{0,11,15-22}$ The release of extracellular vesicles containing Stx (and other virulence factors) by these stimulated cells is considered the trigger producing the transition from bloody diarrhea to life-threatening HUS. ${ }^{23-25}$

Stx activate the innate immune responses, resulting in immunopathologic changes related to inflammation and complement activation. ${ }^{26}$ The multiple interactions of Stx with circulating cells, and with target epithelial and endothelial cells, induce the release of proinflammatory cytokines and chemokines capable of up-regulating the expression of specific toxin receptors (see the next paragraph), of exacerbating tissue damage in targeted organs, and of recruiting inflammatory cells in injured tissues. ${ }^{26}$ Moreover, the activation of the alternative pathway of the complement system by Stx, as part of the innate immune response, is considered a crucial point in the development of renal injuries. ${ }^{26,27}$

\section{Neutral Glycolipids and Proteins as Stx Receptors}

Stx are bipartite exotoxins composed of a single A chain, which is endowed with the enzymatic activity, noncovalently bound to five $\mathrm{B}$ chains forming a pentameric ring that surrounds the $\mathrm{COOH}$-terminus of the A subunit. ${ }^{1,7}$ The latter is a proenzyme that is enzymatically cleaved into two fragments (A1 and A2) linked by a disulfide bond that is reduced within cells, permitting the A1 fragment to express its deadenylating activity on $28 \mathrm{~S}$ rRNA in ribosomes and on
DNA in chromatin, leading to irreversible arrest of translation and to formation of nuclear apurinic sites, respectively. ${ }^{1,7}$ Cleavage can occur during retrograde internalization in target cells by the protease furin or can be induced by extracellular or bacterial proteases. ${ }^{1,7}$ It has been recently shown that cleaved unreduced Stx have changed their blood binding properties with respect to whole toxins. ${ }^{28}$ Stx-intoxicated endothelial cells not only have impaired translation, but also activate a wide array of responses to injury, which have a great impact in the pathogenesis of HUS (eg, release of proinflammatory cytokines, ${ }^{29}$ up-regulation of adhesion molecules, ${ }^{30}$ and activation of the apoptotic program). ${ }^{31}$

Receptor-dependent toxicity is a key concept explaining why these powerful toxins cause damages to selected tissues in animals and humans. The B pentamers of the human subtypes Stx1a and Stx2a show a specific and preferential binding to globotriaosylceramide (Gb3Cer), a member of globoseries glycosphingolipids. ${ }^{1,32}$ The same subtypes also interact, although weakly, with globotetraosylceramide. ${ }^{1,32}$ On the other hand globotetraosylceramide is preferred by the Stx2e subtype associated with edema disease in pigs, that is capable of binding to Gb3Cer, globopentaosylceramide, and Forssman antigen. ${ }^{1}$ Therefore, the tissue expression of these glycolipids and their relative amounts might affect the sensitivity of cells to specific Stx subtypes.

In humans, during HUS, several organs can be involved, with kidney and brain being predominantly targeted. ${ }^{1,2,4}$ The focused expression of Gb3Cer by intestinal, renal, and cerebral endothelial cells and by other cells in the kidney (mesangial cells and tubular and glomerular epithelial cells) allows the toxin to target these organs preferentially by means of multivalent interactions of the glycolipid receptors with B subunits. ${ }^{1,32}$ This is a crucial point to be considered because any hypothesis on the pathogenesis of HUS must explain the focused action of Stx on target cells expressing Gb3Cer. ${ }^{1}$

Besides Gb3Cer-expressing target cells, circulating erythrocytes, monocytes, and platelets also express this receptor with similar lipoforms. ${ }^{1,33}$ Human neutrophils are peculiar in this respect because they specifically bind Stx even though they lack the set of enzymes necessary for the synthesis of these neutral glycolipids. ${ }^{1,34}$ In fact, they are not targeted by Stx; rather, they recognize these bacterial toxins through tolllike receptor 4 (TLR4). ${ }^{35}$ Interestingly, TLR4 interacts with Stx A chain rather than with the B chains. ${ }^{36}$ Moreover, in humans, the receptor pattern of circulating cells is complicated by the simultaneous expression of Gb3Cer and TLR4 by monocytes and platelets. ${ }^{1,33}$

\section{Soluble Factors Present in Blood Affect Stx Activity}

A negative modulator of Stx2, known as human serum amyloid P (HuSAP) component, present in humans, ${ }^{37,38}$ 
binds tightly to this toxin type $\mathrm{e}^{38,39}$ and impairs the Gb3Cerrelated cytotoxic activity. ${ }^{39}$ On the other hand, HuSAP fails to inhibit and rather stimulates Stx2 binding to TLR4expressing human polymorphonuclear leukocytes. ${ }^{40}$ Soluble decoy forms of TLR4 have been described ${ }^{41}$ deriving from alternative mRNA splicing or conversion of the internalized membrane receptor by proteolysis and subsequent release of the truncated form. ${ }^{41,42}$ This is useful in preventing excessive stimulation of innate immunity cells by lipopolysaccharide via TLR $4 .{ }^{43}$ Stx2a interacts with soluble TLR4 in vitro, and the formation of the complex Stx2a/soluble TLR4 allows the toxin to escape capture by HuSAP and to damage Gb3Cer-expressing human cells. ${ }^{44}$ Therefore, a similar protecting interaction of Stx 2 with the extracellular domain of membrane-anchored TLR4 can be expected. The implications of these Stx2-modulating activities on the pathogenesis of HUS are discussed in the following paragraphs.

\section{Extracellular Vesicles Bearing Stx and Their Parent Cells}

In STEC-infected patients, Stx come in contact with and bind to platelets ${ }^{21}$ and leukocytes, and erythrocytes during early toxemia, ${ }^{10,11,16-18,22}$ thereby leading to their activation. ${ }^{20}$ This impacts the formation of circulating plateletmonocyte and platelet-neutrophil aggregates containing Stx, as observed in children with HUS during the acute phase of disease, but not after recovery. ${ }^{16}$ Experimental models with normal whole blood treated with Stx2 and/or STEC-lipopolysaccharide confirm these findings. ${ }^{16}$ Under normal conditions, platelets and leukocytes do not interact in the circulation. Hence, the formation of aggregates between these blood components in STEC-infected patients could play an important role in thrombogenesis and inflammation.

The second more important consequence is the generation of blood cell-derived extracellular vesicles bearing Stx detected in plasma of patients during overt HUS by flow cytometry and visualized within renal endothelial cells by electron microscopy. ${ }^{24}$ The toxins within or on the surface of host blood cell-derived extracellular vesicles can be transferred to target endothelial cells, as shown in in vitro studies (ie, extracellular vesicles containing Stx and derived from human platelets and leukocytes undergo endocytosis in human glomerular endothelial cells and release the toxin, leading to inhibition of protein synthesis and cell death). ${ }^{24}$

The extracellular vesicles involved in the pathogenesis of HUS, analyzed and characterized by transmission electron microscopy, exhibit a rounded shape and $1 \mu \mathrm{m}$ diameter. ${ }^{24}$ This is an important point because this technique allows direct visual inspection of the vesicles, giving a more accurate measure of the size compared with other methods, such as flow cytometry or nanoparticle tracking. ${ }^{45,46} \mathrm{~A}$ weak point of transmission electron microscopy is that it is difficult to ascertain whether the detected vesicles are typical or rare structures. Therefore, it is recommended to supplement transmission electron microscopy with an additional method, allowing detection and analysis of the whole vesicle population.

Besides apoptotic bodies, the other known extracellular vesicles may play a pivotal role in intercellular communication, providing receptors and soluble molecules (mRNA, lipids, and proteins) to recipient cells. ${ }^{45,46}$ The classification of extracellular vesicles is based on their size and structure and on the mechanism of generation. ${ }^{46}$ Microvesicles are shedding vesicles released directly from the plasma membrane by budding, and their diameter (100 to $1000 \mathrm{~nm}$ ) is generally greater than that of exosomes. ${ }^{23,46}$ The latter are small vesicles (30 to $100 \mathrm{~nm}$ ) formed by budding into early endosomes and multivesicular bodies. ${ }^{23,46}$ Exosomes are released by fusion of the external membrane of multivesicular bodies with the plasma membrane. ${ }^{23,46}$ According to the above-described findings, the extracellular vesicles involved in the pathogenesis of HUS more closely resemble microvesicles. ${ }^{24}$

An analysis of the features of the extracellular vesicles found in HUS patients and of the circulating parent cells that release them is crucial to understand their contribution in the pathogenesis of HUS. In a 2-year-old child, platelets (7.9 fL), ${ }^{47}$ erythrocytes (78 fL), ${ }^{48,49}$ neutrophils (299 fL), ${ }^{50,51}$ and monocytes $(355 \mathrm{fL})^{52}$ greatly differ in size. Considering the size of HUS-related rounded $1-\mu \mathrm{m}$-diameter microvesicles $(0.52 \mathrm{fL})$, the volumetric ratio of microvesicle/parent cell is roughly in the range 1:150 to 1:700 for all of the cells while platelets show a 1:15 ratio. Therefore, the number of extracellular vesicles potentially released by a single thrombocyte appears to be limited by the small size of these cell fragments deriving from megakaryocytes. Moreover, the different blood half-lives of erythrocytes (months), platelets (few days), classic monocytes (1 day), and neutrophils (few hours) also affect the daily availability of new vesicle-generating cells entering in circulation from bone marrow.

Taking into account the pediatric blood count ranges of circulating cells ( 1 to 4 years; Mayo Clinic reference value, http://al.mayomedicallaboratories.com/webjc/attachments/ 110/30a2131-complete-blood-count-normal-pediatric-val ues.pdf, last accessed February 19, 2021), ${ }^{47,49,53-56}$ the ratio between the number of vesicles found in the blood of 13 HUS patients (median age, 3 years) ${ }^{24}$ to the number of vesicle-generating cells is maximal for monocytes ( 0.4 to 1.3 vesicles per cell), intermediate for neutrophils ( 4 to $22 \times 10^{-2}$ vesicles per cell), low for platelets ( 2.7 to $3.1 \times 10^{-3}$ vesicles per cell), and extremely low for erythrocytes (7 to $9 \times 10^{-5}$ vesicles per cell). Only a small percentage of microvesicles are Stx2-positive [ie, approximately $25 \%$ to $30 \%$, except erythrocytes $(9 \%)] .{ }^{24}$ The calculated number for neutrophils is probably overestimated because of the known neutrophilia found in HUS patients. ${ }^{2}$ The steady-state blood count of extracellular vesicles is 
determined by a balance between their biogenesis and their delivery to target cells. Nevertheless, when whole blood samples from healthy donors (normal adult blood count ranges; Royal Wolverhampton Trust Pathology Services, https://www.royalwolverhampton.nhs.uk/services/servicedirectory-a-z/pathology-services/departments/haematology/ haematology-normal-adult-reference-ranges, last accessed February 19, 2021) or isolated red cells were treated in vitro with Stx2 in the absence of recipient target cells, similar values were obtained (monocytes, 0.3 to 1.1 vesicles per cell; neutrophils, 2 to $9 \times 10^{-2}$ vesicles per cell; platelets, 1.8 to $5.5 \times 10^{-3}$ vesicles per cell; erythrocytes, $1 \times 10^{-4}$ vesicles per cell). Therefore, although a number of platelet-derived microvesicles prevail in patients' blood during overt HUS, the efficiency of vesicular shedding shown by these cell fragments is lower than that of monocytes and neutrophils. The same ranking in vesicle generation efficiency among human circulating cells was found when unstimulated human blood was analyzed by imaging flow cytometry (ImageStream, Amnis Corporation, Seattle, WA) after simultaneous staining of circulating cells and cell-derived vesicles, ${ }^{45}$ excluding any interference caused by microvesicle isolation procedures in the data obtained with patients' blood. In conclusion, Stx2 enhances the basal level of microvesicle release by circulating cells but does not selectively change the vesiclegenerating ability of a specific circulating cell. Besides Gb3Cer, TLR4 appears to contribute to vesicle generation by interacting with Stx, as demonstrated in Gb3Cer-lacking human neutrophils. ${ }^{24}$

\section{Extracellular Vesicles Bearing Stx Contain Further Virulence Factors Involved in the Pathogenesis of HUS}

Leukocyte-platelet aggregates and microvesicles involved in HUS contain additional pathogenic factors apart from Stx. The deposition of $\mathrm{C} 3$ and $\mathrm{C} 9$ on the surface of circulating blood cell-derived microvesicles, originated from platelets, monocytes, and neutrophils, was observed in the plasma of 12 HUS patients in the acute phase. ${ }^{25}$ Accordingly, C3 bound to platelet-leukocyte complexes (on $30 \%$ of plateletmonocyte aggregates and on 15\% of platelet-neutrophil aggregates) was demonstrated in a 3-year STEC-infected patient with HUS. ${ }^{25}$ These findings have been confirmed in experimental models with whole blood stimulated with Stx and/or STEC-lipopolysaccharide, showing that these virulence factors induced the deposition of surface-bound C3 and C9 on platelet-leukocyte aggregates and derived microvesicles. ${ }^{25}$

In whole blood from four children with HUS, circulating platelet-monocyte and platelet-neutrophil aggregates expressing surface-bound tissue factor (TF) were observed during the acute phase of disease but not after recovery. ${ }^{16} \mathrm{In}$ plasma from the same HUS patients, levels of functional TF and TF-expressing microparticles were also considerably higher than those in pediatric controls. ${ }^{16}$ In in vitro experiments, Stx2 and/or STEC-lipopolysaccharide induced TF expression on platelet-monocyte aggregates compared with phosphate-buffered saline-treated samples. ${ }^{16}$ In addition, an increased number of TF-positive microparticles derived from platelets or monocytes was observed. ${ }^{16}$

The contribution of complement factors and TF to the thrombotic microangiopathy of HUS is clear-cut. Activated complement factors fixed on the surface of extracellular vesicles can be transferred to endothelial target cells, causing injuries. Stx 2 activates complement alternative pathway in the fluid phase and binds to factor $\mathrm{H}$, thereby reducing its protective effect on cellular membranes. ${ }^{27}$ In this respect, it is important to stress that a minority of HUS cases (atypical HUS) are related to genetic defects in regulatory molecules of the complement pathway. ${ }^{1,7}$ TF carried by extracellular vesicles and delivered to target endothelial cells can bind and activate coagulation factor VII, leading to a cascade of reactions culminating in thrombin generation and thus moving the scale toward a prothrombotic state.

\section{Pathotype of HUS-Triggering Extracellular Vesicles}

An emerging concept in microbiology of STEC infections is that of pathotype. To describe strains commonly associated to HUS, it is recommended to refer to the cocktail of virulence factors involved in pathogenesis rather than to the E. coli serogroup, such as $\mathrm{O} 157$ or O26. It is becoming clear that the specific host clinical symptoms depend on the peculiar combinations of virulence factors (pathotype) produced by a specific STEC strain as well as on their allelic types. ${ }^{57}$ Likewise, extracellular vesicles involved in the pathogenesis of HUS should not be considered, based on the type of generating cells, as neutrophil-, monocyte-, or platelet-derived microvesicles nor based on the absolute blood count. Paradoxically, a specific cell-derived microvesicle can have the highest blood count in HUS patients because of delivery systems for target cells. On the other hand, a sparsely represented circulating microvesicle can be promptly cleared from the blood by interactions with target cells. Although the parent cell may confer some of its features to the microvesicles, the specific combination of virulence factors (pathotype) present in the microvesicle/s mainly responsible for the transition from bloody diarrhea to HUS in STEC-infected patients should be investigated. HUS-triggering microvesicles are expected to bear TF, complement factors, and Stx 2 on their surface and within the vesicle as toxic cargo. It is conceivable that the most poisoning HUS-triggering extracellular vesicle/s would have a golden ratio among the different necessary and interactive virulence factors, allowing targeting and full expression of the pathologic effects. 


\section{Is Cerebral Endothelium Differently Targeted in HUS?}

Central nervous system involvement in STEC-infected patients and the role of Stx in this process are intriguing. Neurologic symptoms are more closely related to the direct action of Stx in the brain rather than to the consequences of renal failure (uremic encephalopathy), as demonstrated by their appearance before overt HUS in $15 \%$ of patients. ${ }^{58}$ The underlying mechanisms could be thrombotic microangiopathy in the brain (weak evidence), direct toxic action of Stx 2 on neural cells after blood-brain barrier disruption, or indirect inflammatory-mediated damage to the brain. ${ }^{58}$ Patients with an elevated toxic cargo on neutrophils during the acute phase of HUS are more prone to develop neurologic complications, ${ }^{11,17,33}$ whereas HUS ensues in STEC-infected patients during the toxin-decreasing phase on circulating cells, as demonstrated by the reduced toxic cargo on neutrophils in patients with the most severe forms of HUS. ${ }^{11}$ It can be hypothesized that higher amounts of blood Stx are necessary to induce sudden brain endothelial injuries before the toxin-decreasing phase on circulating cells or that the mechanisms of endothelial damage by Stx are not related to microvesicle release by circulating cells. This interesting topic needs to be further investigated, keeping in mind that fenestrated endothelial cells (renal glomeruli) and those composing the robust blood-brain barrier (brain) differ considerably.

\section{The Observation of STEC-Infected Patients before HUS Provides a Higher Vantage Point}

The important observations described in the previous paragraphs on the role of extracellular vesicles in the pathogenesis of HUS have been obtained after the diagnosis of HUS. ${ }^{16,24,25}$ A recent study analyzed the journey of Stx in the blood of 20 STEC-infected patients after the onset of the prodromal intestinal symptoms (bloody diarrhea) and before the development of HUS. ${ }^{18}$ In this setting, the recognized virulence factors act during the evolution of the pathogenetic process, before the end point (HUS). Contrary to studies on patients in the acute phase of HUS in whom free Stx are rarely found in serum, ${ }^{10-14}$ STEC-infected patients with bloody diarrhea show free Stx in blood detected by enzyme-linked immunosorbent assay (at $\mathrm{ng} / \mathrm{mL}$ concentrations) and by functional assay on human cells (inhibition of protein synthesis). ${ }^{18}$ Few patients develop HUS (15\%); therefore, the mere presence of free Stx in serum (100\% of patients) is not a sufficient condition for the development of the syndrome. Consistently, Lopez et al, ${ }^{59}$ by analyzing about 100 patients with STEC-associated gastroenteritis, found Stx 2 in blood by enzyme-linked immunosorbent assay in patients who developed HUS and in some who recovered. According to other studies on HUS patients, ${ }^{10,11,17,24}$ in most children, Stx were also found by flow cytometry on the surface of the analyzed circulating cells (neutrophils and monocytes). ${ }^{18}$ A quantitative investigation of neutrophil-associated Stx2 obtained by flow cytometry $\left(0.2\right.$ to $1.7 \times 10^{-9} \mathrm{~mol} / \mathrm{L}$, see equation below $)$ or of cell-free circulating Stx 2 detected by enzyme-linked immunosorbent assay $\left(3.3 \text { to } 8.9 \times 10^{-11} \mathrm{~mol} / \mathrm{L}\right)^{18}$ points to the presence of a huge reservoir of cell-associated toxic molecules ready for delivery.

$$
\frac{A \times B \times E}{D \times C}=\text { molarity of Stx } 2 \text { bound to neutrophils }
$$

$\mathrm{A}=2.1 \times 10^{5}$ number of $\mathrm{Stx} 2$ receptors per neutrophil. ${ }^{60}$

$\mathrm{B}=3.5 \times 10^{9}$ pediatric neutrophil count per liter, 2 years, mean. ${ }^{53}$

$\mathrm{C}=3.3 \mathrm{Stx} 2$ bound to neutrophils at saturation, mean channel value of fluorescence. ${ }^{61}$

$\mathrm{D}=6.022 \times 10^{23}$ Avogadro's number.

$\mathrm{E}=0.6$ to $4.5 \mathrm{Stx} 2$ bound to neutrophils in STECinfected patients, mean channel value of fluorescence range. ${ }^{18}$

Kinetic analyses shows that the toxin amounts in circulating leukocytes decrease over time with an increase in the concentration of functionally active Stx in patients' sera, as well as that of the soluble decoy TLR4. ${ }^{18}$ Given the large excess of circulating Stx2-neutralizing HuSAP $\left(10^{-7} \mathrm{~mol} /\right.$ L) ${ }^{37}$ over cell-free Stx2 (see above), the toxicity found in patients' sera can be ascribed to Stx2/TLR4 complexes or to microvesicle-associated Stx2. Furthermore, detachment of Stx from leukocytes was also observed in patients who recovered (even showing proteinuria); thereby, this process, although representing the first step in the delivery to target organs, is not sufficient per se to trigger HUS. ${ }^{18}$

The distinctive feature observed in patients who developed HUS was the sudden appearance of the vesicular form of Stx2 detected by enzyme-linked immunosorbent assay (capturing antibody against B-chains) in blood the day before the onset of HUS during the decreasing phase of toxin on leukocytes. ${ }^{18}$ As with antigens, vesicular toxin was obtained by centrifuging patients' sera at the $g$-force required to isolate $1-\mu \mathrm{m}$ microvesicles and was labeled particulate toxin in contrast to free soluble serum toxin. In these patients, the particulate toxin peak was accompanied by a corresponding peak of particulate TLR4 (microvesicleassociated TLR4), having a stoichiometry ratio with particulate Stx2 close to $1 .^{18}$

The amount of Stx associated with the circulating cells of the patients can decrease over time because of direct transfer of the toxic cargo to Gb3Cer-expressing recipient cells, as observed experimentally during neutrophil transmigration across endothelial monolayers ${ }^{62}$ or release of free Stx (cleared by HuSAP), Stx-TLR4 complex (escaping HuSAP), or microvesicle-associated Stx (within vesicles or Gb3Cer and TLR4 bound). Given the low vesicle generation efficiency of circulating cells (maximal for monocytes, approximately 1 vesicle per cell) and the small amount of 


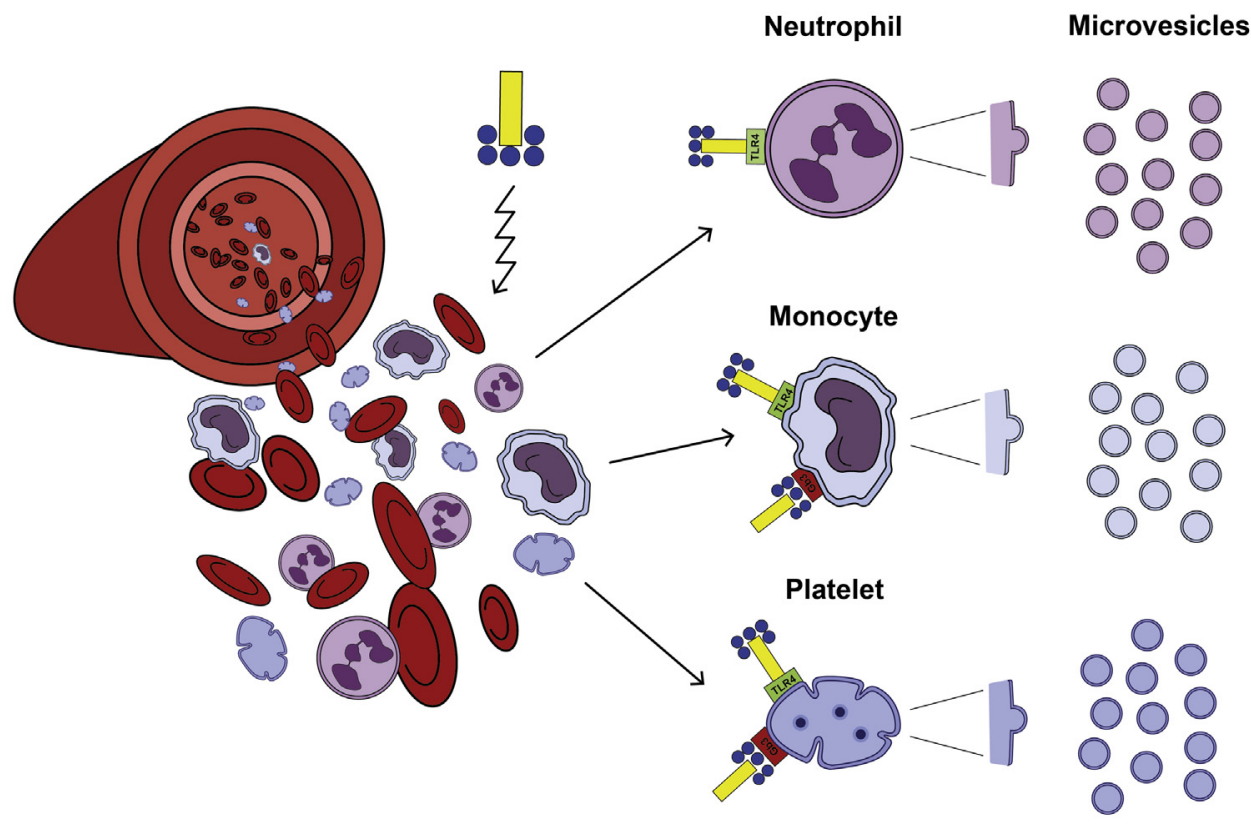

Figure 1 Snapshot of the cells challenged by Shiga toxins (Stx) in the blood of Shiga toxin-producing Escherichia coli (STEC)-infected patients and of their toxin-specific receptors. Stx (left) consisting of a single A chain (yellow rectangle) and five B chains (blue circles) interact with (zigzag arrow) blood circulating cells and platelets through two different receptors: globotriaosylceramide (Gb3Cer; red rectangle), present on monocytes and platelets; and Tolllike receptor 4 (TLR4; green rectangle), present on monocytes, platelets, and neutrophils. The Gb3Cer/Stx B chain and TLR4/Stx A chain interactions on the surface of circulating cells and platelets are shown. A dangerous consequence of these multiple interactions is the budding of microvesicles from Stx-activated cells (right).

microvesicles found in patients' blood, ${ }^{18,24}$ it is unlikely that a sudden decrease in the circulating toxic reservoir could be ascribed to vesicle generation alone. Despite this, there is a good correlation in patients between the toxic activity (translation inhibition in cells) of serum Stx2 and the amount of particulate toxin, but not of whole serum toxin (free toxin + particulate toxin). ${ }^{18}$ Therefore, microvesicleassociated Stx2 stands out as the most ominous toxin form $^{16,24,25}$ found in patients' blood during the transition from bloody diarrhea to HUS. ${ }^{18}$

\section{A Receptor-Based Model for the Specific Targeting of Gb3Cer-Expressing Endothelial Cells by Stx2-Containing Microvesicles}

The role of microvesicles in the pathogenesis of HUS was first explained by the specific binding of Stx 2 to circulating monocytes, platelets, and red cells through Gb3Cer followed by a release phase and a targeting phase. ${ }^{23}$ Extracellular vesicles are capable of interacting with target cells in several ways (ie, binding and dissociation, stable binding, fusion, endocytosis, or receptor-mediated endocytosis). ${ }^{23,46}$ In this model, the toxic cargo, membrane-bound and internalized Stx2, and the associated pathogenic factors, TF and activated complement components, reach the target cells through non-receptor-mediated endocytosis or fusion. Both uptake mechanisms are largely unspecific; therefore, this model failed to explain why the histopathologic lesions observed in HUS patients are focused on few organs and tissues whose cells express Gb3Cer. ${ }^{1,2,4,5}$ HUS is not considered a multi-organ disease. On the other hand, non-receptor-mediated uptake mechanisms have been invoked to explain why other organs apart from intestine, kidney, and brain are affected, albeit rarely, by the thrombotic microangiopathy (eg, pancreas, musculoskeletal system, and heart). ${ }^{1,4}$

Receptor-mediated uptake, favored by mutual molecular interactions between extracellular vesicles and recipient cells, is well-described. ${ }^{23,46}$ In this case, the specific targeting represents the main advantage. Stx associated with microvesicles are primarily localized within the microvesicles because permeabilization is required for maximal detection $^{24}$; nevertheless, the capture of Stx2-containing pathogenic microvesicles circulating in the blood of STEC-infected patients by means of monoclonal antibodies to Stx 2 has been described. ${ }^{18}$ This means that at least part of the toxin is surface bound. It is worth noting that Stx interplay with circulating cells is mediated by two different receptors (Gb3Cer and TLR4), which specifically engage two different parts of the bipartite AB5 toxin (Figure 1). ${ }^{1,35}$ If Stx are bound to microvesicle surface through the glycolipid receptor, their B chains are engaged and presumably the A chain is exposed. Conversely, when the toxins are bound through TLR4 via A chain, the B subunit pentamer is available for specific binding to target cells. Typically microvesicles captured by B-chain specific antibodies are the ones found in the blood of patients the day 


\section{Blood-derived microvesicles}

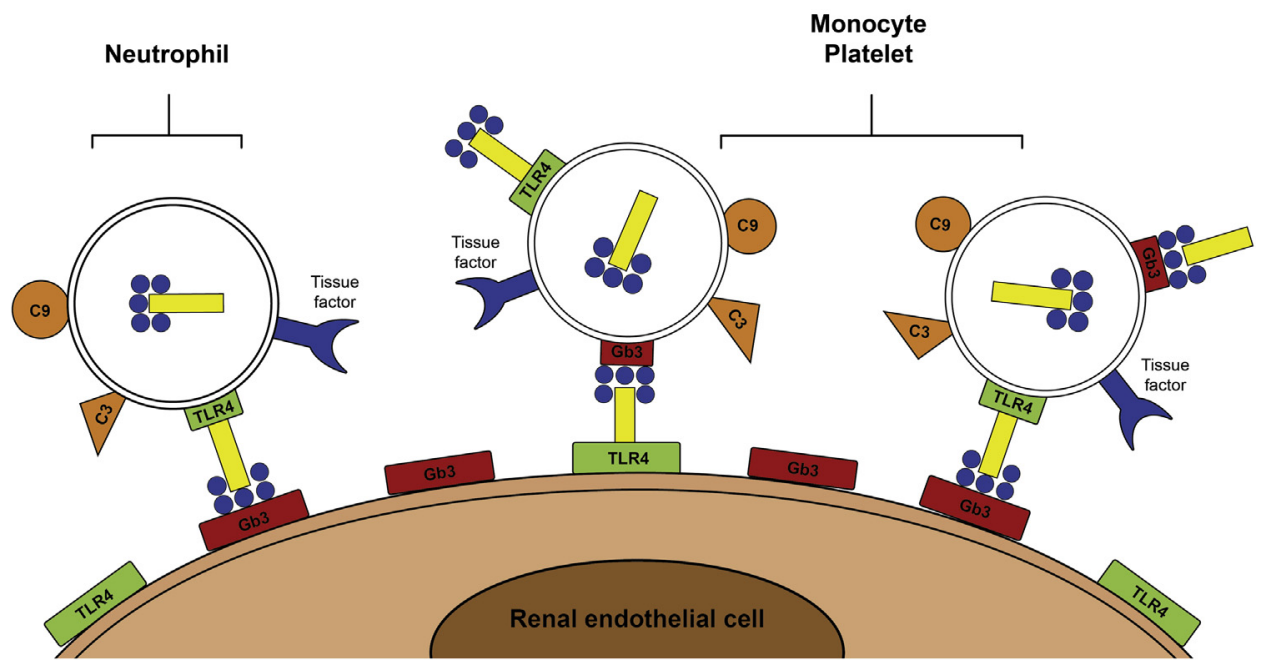

Figure 2 Snapshot of the microvesicles released by platelets, monocytes, and neutrophils after Shiga toxins (Stx) challenge and of their interactions with renal endothelial cell. Microvesicles are endowed with pathogenic factors, such as complement factors 3 (orange triangle) and 9 (orange circles) and tissue factor (blue cup), besides Stx present within and on the surface of the vesicles. Stx A chain (yellow rectangle) binds to Toll-like receptor 4 (TLR4; green rectangle), whereas Stx B chains (blue circles) interact with globotriaosylceramide (Gb3Cer; red rectangle). These receptors are present on microvesicle surface and expressed by target endothelial cells. Targeting of Gb3Cer-expressing endothelial cells by Stx and/or Stx associated with microvesicles is considered the key event in the pathogenesis of hemolytic uremic syndrome. Docking of microvesicles to renal endothelial cells depends on the nature of microvesicle (neutrophil or monocyte/platelet derived) and on the Stx chain involved in the interaction with microvesicle receptors. Monocyte/platelet-derived microvesicles can engage endothelial Gb3Cer through the B chains of TLR4-anchored Stx and endothelial TLR4 through the A chain of Gb3Cer-anchored Stx. Only the former mechanism occurs in neutrophil-derived Gb3Cer-lacking microvesicles. The initial interaction of microvesicles with the endothelial surface is the basis for the successful release of the toxic cargo (Stx and other pathogenic factors) to target cells.

before the fulfillment of the criteria for HUS diagnosis. ${ }^{18}$ These findings are sustained by results obtained in vitro, suggesting that uptake of Stx2-positive microvesicles is not sufficient to induce cell death and that the recipient cell needs to possess Gb3Cer for the the toxin to exert its toxic effect. ${ }^{63}$ The binding of Stx 2 to membrane-associated TLR4 on microvesicles would prevent the interaction of the toxin to HuSAP, a specific inhibitor of the Gb3Cer-dependent toxicity of Stx2, hence favoring the docking to Gb3Cerexpressing renal endothelial cells.

The features of the microvesicles derived from platelets or monocytes are similar because these cells are endowed with both Stx2-interacting receptors (Figure 2). This means that both TLR4-anchored B pentamer-exposing Stx 2 and Gb3Cer-anchored A chain-exposing Stx2 are present on the vesicle surface. Renal endothelial cells also express both these receptors, and when they are targeted by soluble Stx in vitro, TLR4 acts as coreceptor, hence cooperating with Gb3Cer during binding and intoxication. ${ }^{64}$ In this light, the presence of surface-bound Stx2 exposing A or B chains alternatively would confer an advantage to the microvesicle in intoxicating the target cell. Finally, microvesicles derived from neutrophils expose on their surface only the TLR4anchored B pentamer-exposing Stx2.

In conclusion, although the pathogenic cargo depends on the presence, amount, and specific proportion of the different virulence factors associated with the microvesicle, the delivering process is likely to be determined by the presence and spatial orientation of Stx2 exposed on the vesicle membrane. Similar to delivering a package, the delivery of Stx2 to target organs through microvesicles requires a stamp, a price (binding to blood cells); an address, (TLR4-anchored B pentamer-exposing Stx2); and a post box, that is Gb3Cer on target cells. Indeed, Stx are toxic components of the package as well as the leading factors necessary for the docking of the other virulence factors to renal endothelial cells.

This review focuses on the involvement of blood cell- or platelet-derived microvesicles in early pathogenesis of HUS (ie, the initial interactions of virulence factors, including Stx, with renal endothelial cells). In vitro experiments point to the involvement in HUS not only of microvesicle but also of Stx-containing exosomes released by target cells (Vero or HeLa cells) or innate immune cells (macrophage-like differentiated THP-1 cells) after toxin internalization and intracellular processing. ${ }^{65,66}$ Recipient cells bind and internalize Stx-containing exosomes through Gb3Cer receptors because of the presence of Stx on exosome surface. ${ }^{65,66}$ If confirmed in HUS patients, this might be an important mechanism for toxin spreading once the first cells have been targeted through the microvesicle-centered mechanisms reviewed herein. Moreover, Stx-containing exosomes might confer to adjacent nonintoxicated cells not only the toxic cargo but also ancillary factors that exacerbate tissue 
damage, as mRNAs coding for proinflammatory cytokine and chemokines. ${ }^{65}$

Future research perspectives would include the definition of the pathotype of Stx2-bearing HUS-triggering extracellular vesicles (microvesicles and exosomes) and a direct demonstration of their involvement in the targeting of cerebral endothelia. These results will stimulate the development of new strategies for therapeutic interventions aimed at preventing the transition from bloody diarrhea to HUS in STEC-infected children. Blocking the interactions of Stx with circulating cells or the release of the toxic cargo from the circulating or target cells reservoirs by impairing the budding of pathogenic microvesicles and the release of Stxcontaining exosomes would achieve this goal. Glycovesicles spiked with Gb3 neoglycolipids have been developed, which inhibit the binding of Stx to Gb3Cer. ${ }^{67}$ This novel approach would be useful to prevent the targeting of renal endothelium by microvesicle- or exosome-associated Stx in STEC-infected children.

\section{Acknowledgments}

We thank Dr. Marco Varrone for graphical management of figures and Dr. Christine Betts for critical reading of the manuscript.

\section{References}

1. Detzner J, Pohlentz G, Muthing J: Valid presumption of Shiga toxin-mediated damage of developing erythrocytes in EHECassociated hemolytic uremic syndrome. Toxins (Basel) 2020, 12: 373

2. Tarr PI, Gordon CA, Chandler WL: Shiga-toxin-producing Escherichia coli and haemolytic uraemic syndrome. Lancet 2005, 365: $1073-1086$

3. Stevens MP, Frankel GM: The locus of enterocyte effacement and associated virulence factors of enterohemorrhagic Escherichia coli. Microbiol Spectr 2014, 2. EHEC-0007-2013

4. Bielaszewska M, Karch H: Consequences of enterohaemorrhagic Escherichia coli infection for the vascular endothelium. Thromb Haemost 2005, 94:312-318

5. Richardson SE, Karmali MA, Becker LE, Smith CR: The histopathology of the hemolytic uremic syndrome associated with verocytotoxin-producing Escherichia coli infections. Hum Pathol 1988, 19:1102-1108

6. Arvidsson I, Stahl AL, Hedstrom MM, Kristoffersson AC, Rylander C, Westman JS, Storry JR, Olsson ML, Karpman D: Shiga toxin-induced complement-mediated hemolysis and release of complement-coated red blood cell-derived microvesicles in hemolytic uremic syndrome. J Immunol 2015, 194:2309-2318

7. Menge C: Molecular biology of Escherichia coli Shiga toxins' effects on mammalian cells. Toxins (Basel) 2020, 12:345

8. Koutsoumanis K, Allende A, Alvarez-Ordonez A, Bover-Cid S, Chemaly M, Davies R, De Cesare A, Herman L, Hilbert F, Lindqvist R, Nauta M, Peixe L, Ru G, Simmons M, Skandamis P, Suffredini E, Jenkins C, Pires SM, Morabito S, Niskanen T, Scheutz F, da Silva Felıcio MT, Messens W, Bolton D: Pathogenicity assessment of Shiga toxin-producing Escherichia coli (STEC) and the public health risk posed by contamination of food with STEC. EFSA J 2020, 18:e05967
9. Friedrich AW, Bielaszewska M, Zhang WL, Pulz M, Kuczius T, Ammon A, Karch H: Escherichia coli harboring Shiga toxin 2 gene variants: frequency and association with clinical symptoms. J Infect Dis 2002, 185:74-84

10. Brigotti M, Caprioli A, Tozzi AE, Tazzari PL, Ricci F, Conte R, Carnicelli D, Procaccino MA, Minelli F, Ferretti AV, Paglialonga F, Edefonti A, Rizzoni G: Shiga toxins present in the gut and in the polymorphonuclear leukocytes circulating in the blood of children with hemolytic-uremic syndrome. J Clin Microbiol 2006, 44: 313-317

11. Brigotti M, Tazzari PL, Ravanelli E, Carnicelli D, Rocchi L, Arfilli V, Scavia G, Minelli F, Ricci F, Pagliaro P, Ferretti AV, Pecoraro C, Paglialonga F, Edefonti A, Procaccino MA, Tozzi AE, Caprioli A: Clinical relevance of Shiga toxin concentrations in the blood of patients with hemolytic uremic syndrome. Pediatr Infect Dis J 2011, 30:486-490

12. Caprioli A, Luzzi I, Rosmini F, Pasquini P, Cirrincione R, Gianviti A, Matteucci MC, Rizzoni G; The HUS Italian Study Group: Hemolyticuremic syndrome and Vero cytotoxin-producing Escherichia coli infection in Italy. J Infect Dis 1992, 166:154-158

13. Karmali MA, Petric M, Lim C, Fleming PC, Arbus GS, Lior H: The association between idiopathic hemolytic uremic syndrome and infection by verotoxin-producing Escherichia coli. J Infect Dis 1985, 151:775-782

14. He X, Quinones B, Loo MT, Loos S, Scavia G, Brigotti M, Levtchenko E, Monnens L: Serum Shiga toxin 2 values in patients during acute phase of diarrhoea-associated haemolytic uraemic syndrome. Acta Paediatr 2015, 104:e564-e568

15. Arfilli V, Carnicelli D, Ardissino G, Torresani E, Scavia G, Brigotti M: A rapid and sensitive method to measure the functional activity of Shiga toxins in human serum. Toxins (Basel) 2015, 7: 4564-4576

16. Stahl AL, Sartz L, Nelsson A, Bekassy ZD, Karpman D: Shiga toxin and lipopolysaccharide induce platelet-leukocyte aggregates and tissue factor release, a thrombotic mechanism in hemolytic uremic syndrome. PLoS One 2009, 4:e6990

17. Te Loo DM, van Hinsbergh VW, van den Heuvel LP, Monnens LA: Detection of verocytotoxin bound to circulating polymorphonuclear leukocytes of patients with hemolytic uremic syndrome. J Am Soc Nephrol 2001, 12:800-806

18. Brigotti M, He X, Carnicelli D, Arfilli V, Porcellini E, Galassi E, Tazzari PL, Ricci F, Patfield SA, Testa S, Paglialonga F, Picicco D, Caprioli A, Scavia G, Morabito S, Ardissino G: Particulate Shiga toxin 2 in blood is associated to the development of hemolytic uremic syndrome in children. Thromb Haemost 2020 , 120:107-120

19. He X, Ardissino G, Patfield S, Cheng LW, Silva CJ, Brigotti M: An improved method for the sensitive detection of Shiga toxin 2 in human serum. Toxins (Basel) 2018, 10:59

20. Bitzan M, Richardson S, Huang C, Boyd B, Petric M, Karmali MA: Evidence that verotoxins (Shiga-like toxins) from Escherichia coli bind to $\mathrm{P}$ blood group antigens of human erythrocytes in vitro. Infect Immun 1994, 62:3337-3347

21. Karpman D, Papadopoulou D, Nilsson K, Sjogren AC, Mikaelsson C, Lethagen S: Platelet activation by Shiga toxin and circulatory factors as a pathogenetic mechanism in the hemolytic uremic syndrome. Blood 2001, 97:3100-3108

22. van Setten PA, Monnens LA, Verstraten RG, van den Heuvel LP, van Hinsbergh VW: Effects of verocytotoxin-1 on nonadherent human monocytes: binding characteristics, protein synthesis, and induction of cytokine release. Blood 1996, 88:174-183

23. Karpman D, Stahl AL, Arvidsson I: Extracellular vesicles in renal disease. Nat Rev Nephrol 2017, 13:545-562

24. Stahl AL, Arvidsson I, Johansson KE, Chromek M, Rebetz J, Loos S, Kristoffersson AC, Bekassy ZD, Morgelin M, Karpman D: A novel mechanism of bacterial toxin transfer within host blood cell-derived microvesicles. PLoS Pathog 2015, 11:e1004619 
25. Stahl AL, Sartz L, Karpman D: Complement activation on platelet-leukocyte complexes and microparticles in enterohemorrhagic Escherichia coli-induced hemolytic uremic syndrome. Blood 2011, 117:5503-5513

26. Lee MS, Tesh VL: Roles of Shiga toxins in immunopathology. Toxins (Basel) 2019, 11:212

27. Orth D, Khan AB, Naim A, Grif K, Brockmeyer J, Karch H, Joannidis M, Clark SJ, Day AJ, Fidanzi S, Stoiber H, Dierich MP, Zimmerhackl LB, Wurzner R: Shiga toxin activates complement and binds factor $\mathrm{H}$ : evidence for an active role of complement in hemolytic uremic syndrome. J Immunol 2009, 182:6394-6400

28. Brigotti M, Orth-Holler D, Carnicelli D, Porcellini E, Galassi E, Tazzari PL, Ricci F, Manoli F, Manet I, Talasz H, Lindner HH, Speth C, Erbeznik T, Fuchs S, Posch W, Chatterjee S, Wurzner R The structure of the Shiga toxin 2a A-subunit dictates the interactions of the toxin with blood components. Cell Microbiol 2019, 21:e13000

29. Jandhyala DM, Thorpe CM, Magun B: Ricin and Shiga toxins: effects on host cell signal transduction. Curr Top Microbiol Immunol 2012, 357:41-65

30. Zoja C, Buelli S, Morigi M: Shiga toxin-associated hemolytic uremic syndrome: pathophysiology of endothelial dysfunction. Pediatr Nephrol 2010, 25:2231-2240

31. Tesh VL: The induction of apoptosis by Shiga toxins and ricin. Curr Top Microbiol Immunol 2012, 357:137-178

32. Muthing J, Schweppe CH, Karch H, Friedrich AW: Shiga toxins, glycosphingolipid diversity, and endothelial cell injury. Thromb Haemost 2009, 101:252-264

33. Brigotti M: The interactions of human neutrophils with Shiga toxins and related plant toxins: danger or safety? Toxins (Basel) 2012, 4: $157-190$

34. Macher BA, Klock JC: Isolation and chemical characterization of neutral glycosphingolipids of human neutrophils. J Biol Chem 1980, 255:2092-2096

35. Brigotti M, Carnicelli D, Arfilli V, Tamassia N, Borsetti F, Fabbri E, Tazzari PL, Ricci F, Pagliaro P, Spisni E, Cassatella MA: Identification of TLR4 as the receptor that recognizes Shiga toxins in human neutrophils. J Immunol 2013, 191:4748-4758

36. Arfilli V, Carnicelli D, Rocchi L, Ricci F, Pagliaro P, Tazzari PL, Brigotti M: Shiga toxin 1 and ricin A chain bind to human polymorphonuclear leucocytes through a common receptor. Biochem J 2010, 432:173-180

37. Armstrong GD, Mulvey GL, Marcato P, Griener TP, Kahan MC, Tennent GA, Sabin CA, Chart H, Pepys MB: Human serum amyloid P component protects against Escherichia coli O157:H7 Shiga toxin 2 in vivo: therapeutic implications for hemolytic-uremic syndrome. J Infect Dis 2006, 193:1120-1124

38. Kimura T, Tani S, Matsumoto Yi Y, Takeda T: Serum amyloid P component is the Shiga toxin 2-neutralizing factor in human blood. J Biol Chem 2001, 276:41576-41579

39. Marcato P, Vander Helm K, Mulvey GL, Armstrong GD: Serum amyloid $\mathrm{P}$ component binding to Shiga toxin 2 requires both a subunit and B pentamer. Infect Immun 2003, 71:6075-6078

40. Griener TP, Mulvey GL, Marcato P, Armstrong GD: Differential binding of Shiga toxin 2 to human and murine neutrophils. J Med Microbiol 2007, 56:1423-1430

41. Zunt SL, Burton LV, Goldblatt LI, Dobbins EE, Srinivasan M: Soluble forms of toll-like receptor 4 are present in human saliva and modulate tumour necrosis factor-alpha secretion by macrophage-like cells. Clin Exp Immunol 2009, 156:285-293

42. Jaresova I, Rozkova D, Spisek R, Janda A, Brazova J, Sediva A: Kinetics of toll-like receptor-4 splice variants expression in lipopolysaccharidestimulated antigen presenting cells of healthy donors and patients with cystic fibrosis. Microbes Infect 2007, 9:1359-1367

43. Iwami KI, Matsuguchi T, Masuda A, Kikuchi T, Musikacharoen T, Yoshikai Y: Cutting edge: naturally occurring soluble form of mouse Toll-like receptor 4 inhibits lipopolysaccharide signaling. J Immunol 2000, 165:6682-6686
44. Brigotti M, Arfilli V, Carnicelli D, Ricci F, Tazzari PL, Ardissino G, Scavia G, Morabito S, He X: Soluble toll-like receptor 4 impairs the interaction of Shiga toxin 2a with human serum amyloid $\mathrm{P}$ component. Toxins (Basel) 2018, 10:379

45. Headland SE, Jones HR, D'Sa AS, Perretti M, Norling LV: Cuttingedge analysis of extracellular microparticles using ImageStream $(\mathrm{X})$ imaging flow cytometry. Sci Rep 2014, 4:5237

46. Raposo G, Stoorvogel W: Extracellular vesicles: exosomes, microvesicles, and friends. J Cell Biol 2013, 200:373-383

47. Novak RW, Tschantz JA, Krill CE: Normal platelet and mean platelet volumes in pediatric patients. Lab Med 1987, 18:613-614

48. Dallman PR, Siimes MA: Percentile curves for hemoglobin and red cell volume in infancy and childhood. J Pediatr 1979, 94:26-31

49. Saarinen UM, Siimes MA: Developmental changes in red blood cell counts and indices of infants after exclusion of iron deficiency by laboratory criteria and continuous iron supplementation. J Pediatr 1978, 92:412-416

50. Rosenbluth MJ, Lam WA, Fletcher DA: Force microscopy of nonadherent cells: a comparison of leukemia cell deformability. Biophys J 2006, 90:2994-3003

51. Ting-Beall HP, Needham D, Hochmuth RM: Volume and osmotic properties of human neutrophils. Blood 1993, 81:2774-2780

52. Downey GP, Doherty DE, Schwab B 3rd, Elson EL, Henson PM, Worthen GS: Retention of leukocytes in capillaries: role of cell size and deformability. J Appl Physiol (1985) 1990, 69:1767-1778

53. Dallman PR. Edited by Rudolph's Pediatrics. ed 16. New York: Appleton-Century-Crofts, 1977

54. Lanzkowsky P. Edited by Pediatric Hematology-Oncology, a Treatise for the Clinician. New York: McGraw-Hill, 1980

55. Miller DC. Edited by Blood Diseases of Infancy and Childhood. ed 5. St. Louis, MO: Mosby, 1984

56. Nathan DG, Oski FA. Hematology of Infancy and Childhood. ed 3. Philadelphia, PA: Saunders, 1987

57. Karmali MA, Gannon V, Sargeant JM: Verocytotoxin-producing Escherichia coli (VTEC). Vet Microbiol 2010, 140:360-370

58. Goldstein J, Nunez-Goluboay K, Pinto A: Therapeutic strategies to protect the central nervous system against Shiga toxin from enterohemorrhagic Escherichia coli. Curr Neuropharmacol 2021, 19: 24-44

59. Lopez EL, Contrini MM, Glatstein E, Gonzalez Ayala S, Santoro R, Ezcurra G, Teplitz E, Matsumoto Y, Sato H, Sakai K, Katsuura Y, Hoshide S, Morita T, Harning R, Brookman S: An epidemiologic surveillance of Shiga-like toxin-producing Escherichia coli infection in Argentinean children: risk factors and serum Shiga-like toxin 2 values. Pediatr Infect Dis J 2012, 31:20-24

60. te Loo DM, Monnens LA, van Der Velden TJ, Vermeer MA, Preyers F, Demacker PN, van Den Heuvel LP, van Hinsbergh VW: Binding and transfer of verocytotoxin by polymorphonuclear leukocytes in hemolytic uremic syndrome. Blood 2000, 95:3396-3402

61. Tazzari PL, Ricci F, Carnicelli D, Caprioli A, Tozzi AE, Rizzoni G, Conte R, Brigotti M: Flow cytometry detection of Shiga toxins in the blood from children with hemolytic uremic syndrome. Cytometry B Clin Cytom 2004, 61:40-44

62. Brigotti M, Tazzari PL, Ravanelli E, Carnicelli D, Barbieri S, Rocchi L, Arfilli V, Scavia G, Ricci F, Bontadini A, Alfieri RR, Petronini PG, Pecoraro C, Tozzi AE, Caprioli A: Endothelial damage induced by Shiga toxins delivered by neutrophils during transmigration. J Leukoc Biol 2010, 88:201-210

63. Johansson K, Willysson A, Kristoffersson AC, Tontanahal A, Gillet D, Stahl AL, Karpman D: Shiga toxin-bearing microvesicles exert a cytotoxic effect on recipient cells only when the cells express the toxin receptor. Front Cell Infect Microbiol 2020, 10:212

64. Torgersen ML, Engedal N, Pedersen AM, Husebye H, Espevik T, Sandvig K: Toll-like receptor 4 facilitates binding of Shiga toxin to colon carcinoma and primary umbilical vein endothelial cells. FEMS Immunol Med Microbiol 2011, 61:63-75 
65. Lee KS, Lee J, Lee P, Kim CU, Kim DJ, Jeong YJ, Park YJ, Tesh VL, Lee MS: Exosomes released from Shiga toxin 2a-treated human macrophages modulate inflammatory responses and induce cell death in toxin receptor expressing human cells. Cell Microbiol 2020, 22:e13249

66. Watanabe-Takahashi M, Yamasaki S, Murata M, Kano F, Motoyama J, Yamate J, Omi J, Sato W, Ukai H, Shimasaki K, Ikegawa M, Tamura-Nakano $\mathrm{M}$, Yanoshita R, Nishino $\mathrm{Y}$,
Miyazawa A, Natori Y, Toyama-Sorimachi N, Nishikawa K: Exosome-associated Shiga toxin 2 is released from cells and causes severe toxicity in mice. Sci Rep 2018, 8:10776

67. Pohlentz G, Steil D, Rubin D, Mellmann A, Karch H, Muthing J: Pectin-derived neoglycolipids: tools for differentiation of Shiga toxin subtypes and inhibitors of Shiga toxin-mediated cellular injury. Carbohydr Polym 2019, 212:323-333 